\title{
Comparison of Tibial Tuberosity-Trochlear Groove (TT-TG) distances between different anterior cruciate ligament reconstructions: A retrospective clinical and imaging study
}

\author{
Jianfeng Liu ${ }^{\mathrm{a}, 1}$, Junchen Wang ${ }^{\mathrm{b}, 1}$, Liyuan Tao ${ }^{\mathrm{c}, 1}$, Chenlu Liu ${ }^{\mathrm{d}}$, Yongxiang Wang ${ }^{\mathrm{a}}$, \\ Baogang $\mathrm{Wei}^{\mathrm{a}}$, Pengfei $\mathrm{Li}^{\mathrm{a}}$, Huricha $\mathrm{Bao}^{\mathrm{a}}$, Bingxian $\mathrm{Ma}^{\mathrm{a}}$, Yansong $\mathrm{Qi}^{\mathrm{a}, *}$ and Yongsheng $\mathrm{Xu}^{\mathrm{a}, *}$ \\ ${ }^{a}$ Department of Orthopaedics, Inner Mongolia People's Hospital, Hohhot, China \\ ${ }^{\mathrm{b}}$ School of Mechanical Engineering and Automation, Beihang University, Beijing, China \\ ${ }^{\mathrm{c}}$ Research Center of Clinical Epidemiology, Peking University Third Hospital, Beijing, China \\ ${ }^{\mathrm{d}}$ Department of Radiology, Peking University Third Hospital, Beijing, China
}

\begin{abstract}
.
BACKGROUND: Previous studies showed that compared with single-bundle (SB) precedures, double-bundle (DB) anterior cruciate ligament (ACL) reconstruction perform better.

OBJECTIVE: To make assurance that distance of TT-TG may be altered along with ACL rupture and reconstruction. METHODS: Imaging study of 201 patients's related cases by MRI and CT scans.

RESULTS: Compared with the intact knee's overall mean TT-TG value, the mean overall pre/postoperative TT-TG values showed a significant difference. For SB reconstruction, the mean pre/postoperative TT-TG values were $15.67 \pm 2.46 \mathrm{~mm}$ and $14.72 \pm 2.48 \mathrm{~mm}$, respectively. Postoperative and intact knee TT-TG values were significantly different $(p<0.001)$. For DB reconstruction, the pre/postoperative mean TT-TG values were $15.11 \pm 1.99 \mathrm{~mm}$ and $13.11 \pm 1.71 \mathrm{~mm}$. Postoperative and intact knee TT-TG values were not significantly different $(p=0.141)$.

CONCLUSIONS: The increased TT-TG value from a ruptured ACL was significantly restored after ACL reconstruction. The TT-TG value after SB reconstruction was still obviously larger than that of the intact knee. It showed no significant difference between the postoperative TT-TG of the DB group and intact knees. The original TT-TG values of the knees were much closer to restoration after DB reconstruction.
\end{abstract}

Keywords: TT-TG, ACL reconstruction, single-bundle, double-bundle

\section{Introduction}

Providing stability to the knee joint and minimizing stress across knee is the main function of the anterior cruciate ligament (ACL). It restrains excessive forward movement of the tibia and limits rotational movements of the knee $[1,2]$. ACL reconstruction aims to restore normal knee function. Over

\footnotetext{
${ }^{1}$ Equal contributors.

${ }^{*}$ Corresponding authors: Yongsheng Xu, Department of Orthopaedics, Inner Mongolia People's Hospital, Hohhot 010017, China. Tel.: +86 18047192357; E-mail: dlxyf@ 163.com; Yansong Qi, Department of Orthopaedics, Inner Mongolia People’s Hospital, Hohhot 010017, China. Tel.: +86 13789418632; E-mail: malaqinfu@ 126.com.
}

0928-7329/19/\$35.00 (c) 2019 - IOS Press and the authors. All rights reserved

This article is published online with Open Access and distributed under the terms of the Creative Commons Attribution NonCommercial License (CC BY-NC 4.0). 
the past decade, ACL reconstruction has been undergone as a SB reconstruction, which is the gold standard technique worldwide. Recently, however, several scholars have conducted double-bundle (DB) reconstruction [1,3-7]. SB ACL reconstruction does not restore tibial rotation [9-12]. Excessive tibial rotation will lead to abnormal loading of the cartilage areas that leads to osteoarthritis finally [13]. Fu et al. [1] suggested that is an important that restoring knee stability decrease the incidence of degenerative changes through ACL reconstruction after rupture of the ACL. Tajima et al. [14] reported that DB ACL reconstruction restores the patellofemoral joint contact area and contact pressures better than SB ACL reconstruction. Besides, the tibial tuberosity-trochlear groove (TT-TG) distance of an ACL deficit knee may be altered and may require restoration after reconstruction. However, information on this topic is limited. The TT-TG value could indicate the displacement relationship of the tibiofemoral joint and could be used to show tibial rotation stability.

We hypothesized that the TT-TG value may be altered after ACL rupture and reconstruction and that two different surgical methods would produce different TT-TG results.

\section{Materials and methods}

\subsection{Clinical data}

This retrospective study included patients in our hospital from February, 2008 to April, 2016, males and females with a primary one-sided total ACL rupture undergoing arthroscopic SB or DB ACL reconstruction. All arthroscopic reconstructions were conducted by the same senior surgeon (Yong-Sheng $\mathrm{Xu}$ ). All patients involved in this study gave their informed consent. Institutional review board approval of Inner Mongolia People's Hospital was obtained for this study.

Patients with the following conditions underwent SB ACL reconstruction: (1) a small or narrow intercondylar fossa; (2) still at a growing age; (3) severe arthritis; and (4) multiple knee ligament injuries or a knee dislocation. Otherwise, patients underwent DB ACL reconstruction.

Patients meeting the following criteria were excluded: (1) patellofemoral disorder or patellar dislocation; (2) lower limb fractures; (3) active infection affecting the limb subject to treatment/possibilitis could lead to postoperative infection; (4) multiple ligament injuries in the ipsilateral knee, e.g., MCL or PCL injury; (5) meniscal injuries that were addressed with partial resection of more than $>1 / 3$ of the area; (6) knee cartilage injury of grade III; (7) knee degeneration of grade III or above on the KellgrenLawrence scale [15]; (8) general joint laxity with a score of 4 or above on the Beighton-Horan index [16]; (9) contraindications for MRI-scanning; and (10) professional or highly competitive athletes.

Imaging analysis was performed by a senior orthopaedic surgeon (YXW) and an imaging technician (CLL), who measured each other blindly and reported the consistency of the measurements, the intraclass correlation coefficient (ICC) is 0.913 . Preoperative and postoperative image examinations were conducted as follows: (1) MRIs of the injured knee with a scan range from the trochlear groove to the tibial tuberosity were obtained with a fully extended knee for each patient. (2) Patellar lateral projection $\mathrm{X}$-ray images of the injured knee flexed at $30^{\circ} / 60^{\circ} / 90^{\circ}$ were captured to evaluate any patellofemoral disorders. (3) Anterior-posterior and medial-lateral projection X-ray images of the injured knee were captured to evaluate degeneration of the knee. (4) Postoperative CT scans of both knees fully extended were recorded for TT-TG and bone tunnel position measurements. 
Table 1

MRI parameters

\begin{tabular}{lcccc}
\hline & Sag T1 & Sag PD & Cor PD & AxPD \\
\hline Sequence & FSE & FSE & FSE & FSE \\
Fat saturation & No & Yes & Yes & Yes \\
TR $(m s)$ & 779 & 3016 & 2976 & 2125 \\
TE $(m s)$ & Min full & 32 & 32 & 35 \\
Matrix size & $384 \times 256$ & $320 \times 288$ & $320 \times 256$ & $320 \times 288$ \\
FOV $(\mathrm{cm})$ & 16 & 16 & 16 & 16 \\
Thickness $(\mathrm{mm})$ & 3.5 & 3.5 & 3.5 & 4 \\
Space $(\mathrm{mm})$ & 0.5 & 0.5 & 0.5 & 0.8 \\
\hline
\end{tabular}

FSE: fast spin echo, SE: spin echo, TR: repetition time, TE: echo time, FOV: field of view.

\subsection{Operation technique}

Traditional arthroscopic SB ACL reconstruction was conducted with a technique as the same as Harner et al. [17], Staubli and Rauschning [18] and Gong et al. [19] whose techniques previously reported. A semitendinosus-gracilis tendon (STG) graft was selected. The graft was assembled in the same way for the DB reconstruction protocol was previously reported by Yu et al. [5] and Leung et al. [20].

\subsection{Postoperative rehabilitation protocol}

Both SB and DB reconstruction groups adopted the same rehabilitation protocol. Patients began rangeof-motion exercises 4 days after the operation. Postoperative CT scans of both knees and anteroposterior and lateral radiographs were taken 4 days after surgery. After 6 weeks, full weight-bearing with a knee brace was permitted, and the knee should have been able to flex to at least $120^{\circ}$ and extend to the same angle of the bilateral intact knee. Knee brace protection during activities was required until 3 months after surgery, when flexion and extensionreached as same as the intact kneepostoperatively, we encouraged patients to start jogging and swimming. Full physical exercises should be limited in 6 months postoperatively. Postoperative outpatient follow-up visits occurred after 6 weeks, 3, 6, and 12 months, and the follow-up period after one year is every other year.

\section{Measurements}

\subsection{MRI examination protocol}

The MRI protocol was performed as previously reported [21,22]. We adopted a 3.0-Tesla MR system (Magnatom 750WS, GE Medical Systems, USA) using a knee coil (GE Medical Systems, USA) with he following series: (1) sagittal T1-weighted spin echo: repetition time (TR) $779 \mathrm{~ms}$, echo time (TE) Min full ms; (2) sagittal protein density (PD) fast-saturated spin echo: TR $3016 \mathrm{~ms}$, TE $32 \mathrm{~ms}$; (3) coronal PD fat-saturated spin echo: TR $2976 \mathrm{~ms}$, TE $32 \mathrm{~ms}$; and (4) Ax PD fat-saturated fast spin echo: TR $2125 \mathrm{~ms}$, TE $35 \mathrm{~ms}$. All serials included in Table 1.

\subsection{CT examination protocol}

A spiral CT scan (CT Machine: Siemens Medical Systems, Erlangen, Germany) was systematically performed. The patients were placed supine on the scanner and both knees were positioned in neutral rotation. The scanning procedure acquired $4.0 \mathrm{~mm}$ CT slices from the hip joint to the ankle joint (Table 2). 
Table 2

CT parameters

\begin{tabular}{ll}
\hline Factors & \\
\hline $\mathrm{mAs}$ & 100 \\
$\mathrm{kVp}$ & 120 \\
Type of scan & Axial \\
Scan range & Lower extremity \\
Gantry tilt & $0^{\circ}$ \\
Algorithm & Bone \\
Matrix size & $512 \times 512$ \\
Field of view & $18 \mathrm{~cm}$ \\
Calibration file & Large body \\
Thickness (mm) & 4 \\
mA and scan time (mAs) & 100 \\
\hline
\end{tabular}

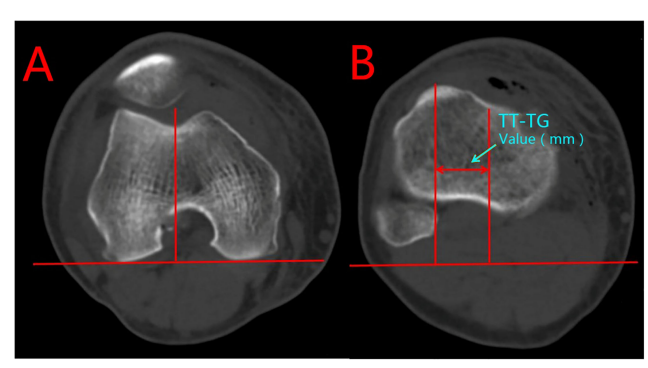

Fig. 1. TT-TG Measurements on CT.

\subsection{TT-TG values}

The postoperative TT-TG values of both SB and DB groups were measured between the most anterior point of the tibial tuberosity and the deepest bony point of the trochlear groove using lines perpendicular to the tangent of the bony borders of the posterior condyles on axial CT scans, as illustrated by Goutallier et al. [23] except that the CT scans were performed with the knee fully flexed. A tangent line was drawn on the axial section passing through the posterior femoral condyles. Next, a perpendicular line was drawn through the deepest point of the trochlea and then transferred to the axial section with the most anterior point of the tibial tuberosity. A line was drawn through the most anterior point of the tibial tuberosity and perpendicular to the baseline. The distance between the two perpendicular lines was measured in millimetres (Fig. 1). A high correlation between the CT scan and MRI scan measurements of the TT-TG were demonstrated by Schoettle et al. [24]. To protect patients from excessive radiation within a short interval, the preoperative TT-TG values were measured from the patients' preoperative MRI scans (Fig. 2). All computerized digital measurements were conducted by one observer using the picture archiving and communications system (PACS) of our department.

\subsection{Statistical analysis}

Statistical analysis was performed with SPSS 25.0 software for Windows (SPSS, Inc., Chicago, IL, USA). A paired t-test was used to compare the pre- and postoperative TT-TG values. The independent sample t-test was used to compare the TT-TG values between two ACL reconstructions groups and intact knees. Significance was established at $p<0.05$ (two-sided). 
Table 3

Patient data

\begin{tabular}{lrccccc}
\hline & \multicolumn{1}{c}{$n$} & Age $(\mathrm{y}) *$ & Male* $^{*}$ & Female* & Left knee* & Right knee* \\
\hline SB group & 103 & $32.6 \pm 3.32$ & 71 & 32 & 31 & 72 \\
DB group & 98 & $33.2 \pm 3.75$ & 63 & 35 & 39 & 59 \\
\hline
\end{tabular}

${ }^{*}$ No significant difference between the patient data of the two groups at $p>0.05$.

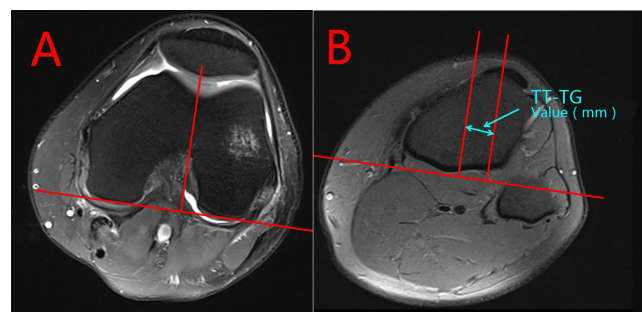

Fig. 2. TT-TG Measurements on MRI.

\section{Results}

\subsection{Patient data}

Overall, 201 patients (134 males and 67 females) with an average age of $32.89 \pm 3.41$ (range: 18-40) years were included in the study. The patient data of the two groups were not significantly different (Table 3). Concurrent with the ACL reconstruction, 17 patients also underwent Fast-fix suturing of the lateral or medial meniscus; 51 patients had a partial meniscectomy of the lateral, medial or bilateral meniscus, and 22 patients underwent shaver debridement or radiofrequency gasification on cartilage lesions.

\subsection{TT-TG values}

The overall mean TT-TG value of the intact knee was $12.41 \pm 2.21 \mathrm{~mm}$. The mean overall preoperative TT-TG value was $15.40 \pm 2.21 \mathrm{~mm}$. The mean overall postoperative TT-TG value was $13.94 \pm 2.18 \mathrm{~mm}$. The overall preoperative and postoperative TT-TG values were significantly different $(p<0.001)$. The mean preoperative and postoperative TT-TG values of the SB group were $15.67 \pm 2.46 \mathrm{~mm}$ and $14.72 \pm$ $2.48 \mathrm{~mm}$, respectively. The postoperative TT-TG value of patients undergoing SB ACL reconstruction was significantly smaller than that before surgery $(p=0.013)$. The postoperative and healthy knee TTTG values $(p<0.001)$ were significantly different. The mean preoperative and postoperative TT-TG values of the DB group were $15.11 \pm 1.99 \mathrm{~mm}$ and $13.11 \pm 1.71 \mathrm{~mm}$, respectively. The TT-TG values of patients who underwent DB ACL reconstruction were also significantly smaller than those before surgery $(p<0.001)$. The postoperative TT-TG values in the DB group and the healthy knee TT-TG values were not significantly different $(p=0.141)$ (Table 4$)$.

\section{Discussion}

Patellofemoral disorder is a common orthopaedic problem. The ACL's function is to stabilize the knee and minimize stress across the knee joint. The ACL restrains excessive forward movement of the tibia 
Table 4

TT-TG values of preoperative, postoperative and intact knee

\begin{tabular}{lrccccrr}
\hline & \multicolumn{1}{c}{$N$} & Intact knee $(\mathrm{mm})$ & Preoperative & Postoperative & $\mathrm{p} 1$ & $\mathrm{p} 2$ & \multicolumn{1}{c}{$\mathrm{p} 3$} \\
\hline Overall & 201 & $12.41 \pm 2.21$ & $15.40 \pm 2.21$ & $13.94 \pm 2.18$ & $<0.001$ & 0.076 & $<0.001$ \\
SB group & 103 & $12.35 \pm 2.52$ & $15.67 \pm 2.46$ & $14.72 \pm 2.48$ & $<0.001$ & $<0.001$ & 0.013 \\
DB group & 98 & $12.45 \pm 1.95$ & $15.11 \pm 1.99$ & $13.11 \pm 1.71$ & $<0.001$ & 0.141 & $<0.001$ \\
\hline
\end{tabular}

Note: $\mathrm{p} 1$ - the statistical significant between the preoperative and the intact TT-TG values, $\mathrm{p} 2$ - the statistical significant between the postoperative and the intact TT-TG values, p3 - the statistical significant between the preoperative and the postoperative TT-TG values.

and limits rotational movements of the knee $[1,2,25]$. Chronic injuries, degeneration of the articular cartilage, meniscal tears and arthritis may be caused by anterior-posterior instability, rotatory instability, etc. could cause ACL deficiency [13]. Stergiou et al. [13] stated that excessive tibial rotation leads to abnormal loading of the cartilage areas that are not commonly loaded in the healthy knee. Over time, this abnormal loading will lead to osteoarthritis. ACL reconstruction aims to restore normal knee function. However, Biau et al. [8] reported that only $41 \%$ and $33 \%$ of patients undergoing SB ACL reconstruction with patellar and hamstring tendon grafts respectively, realize a recovery to a normal state based on the final overall IKDC score. Georgoulis et al. $[10,26]$ demonstrated that ACL reconstruction was not fit to restore tibial rotation totally. As a result, cartilage degeneration still increased after ACL reconstruction, despite SB or DB ACL reconstruction.

Our study showed that the TT-TG values were significantly restored after ACL reconstruction. The restoration of the TT-TG values not only indicated that ACL reconstruction could improve the anteriorposterior stability but also significantly improve rotational knee stability based on tibial rotation. However, the restored TT-TG values of the SB and DB ACL reconstructions were not significantly different.

Our results thus demonstrated that traditional SB ACL reconstruction cannot fully restore the native anatomy of the affected knee. Although the TT-TG values of the patients were partly restored to a closerto-normal level, the intact knee and the SB groups were still significantly different. More specifically, the traditional SB ACL reconstruction could not fully restore the tibial rotation of the knee. Correspondingly, Yu et al. [5] and Tajima et al. [14] have demonstrated that SB ACL reconstruction could not restore the normal pressure area of the patellofemoral joint as well as DB ACL reconstruction.

In DB ACL reconstruction group, the patients' TT-TG values were restored to a level not significantly different from that of an intact knee, while the SB reconstruction did not reach such restoration levels. This may indicate that the DB ACL reconstruction better resembles the native anatomy of the ACL and the native knee kinematics than SB ACL reconstruction. The information from the 1-year postoperative second-look arthroscopy during hardware removal of patients who underwent DB ACL reconstruction with our research team indicated that the articular cartilage degenerated significantly less than that in patients who underwent SB reconstruction, which suggests that DB ACL reconstruction could more significantly decelerate the postoperative cartilage damage than SB ACL reconstruction.

Muneta et al. [3] ran a randomized clinical study and demonstrated that DB ACL reconstruction patients showed a significantly smaller KT-1000 side-to-side and a higher number of negative Lachman and pivot-shift tests. For biomechanical studies, Yagi et al. [4] found that anatomic DB ACL reconstruction could produce a better biomechanical outcome and was significantly closer to the intact knee in cadavers. Morimoto et al. [27] found that SB ACL reconstruction resulted in a smaller tibiofemoral contact area and caused higher pressure, while DB ACL reconstruction could more closely restore the normal contact area and pressure, mainly at low flexion angles. Tashman et al. [11] and Gabriel et al. [9] concluded that SB reconstruction only restores most of the anterior-posterior knee stability, exerting control over most forward tibial shifting, but does not fully restore the stability in tibial rotation during activities with high 
functional demands. These findings indicated that DB ACL reconstruction could more closely restore the anatomy of a normal ACL.

Dejour et al. [28] revealed four anatomical factors related to patellofemoral disorders: trochlear dysplasia, quadriceps dysplasia, patella alta and increased TT-TG distance. A medialized trochlear groove in the case of trochlear dysplasia or a lateralised tibial tuberosity could increase TT-TG distance. The restoration of the TT-TG value to a level not significantly different from that of an intact knee after DB ACL reconstruction in our study could indicated that the rotational and medial-lateral relationship of the tibiofemoral joint was better restored with DB ACL reconstruction than with SB ACL reconstruction.

Hsieh et al.'s study [29], supported by the observation of Shin et al. [30] in an in vivo kinematic study, showed that removal of the ACL might lead to a significant increase in anterior translation and valgus rotation of the tibia and in lateral tilt and lateral shift of the patella. Intra-articular reconstruction could restore these to levels similar to the intact knee in a cadaveric study. The findings of our study showed that the TT-TG value is enlarged in an ACL ruptured knee and is restored after ACL reconstruction, indicating the restoration of tibial rotation. However, during in vivo weight-bearing activity, Van de Velde et al. [31] showed that abnormal patellar rotation, tilt, and lateral shift in cartilage contact persisted after SB ACL reconstruction. These persistent abnormalities of the patellofemoral contact characteristics and motion may be associated with that patellofemoral cartilage degeneration continued to aggravate after ACL reconstruction. We also found that the TT-TG value is not fully restored after SB ACL reconstruction. The relationship between contact characteristics and articular cartilage metabolism is currently unclear. The lateralization of the patella, along with the corresponding changes in the patellofemoral contact area and pressure, may affect articular cartilage adversely. By restoring the TT-TG value to a normal level after DB ACL reconstruction, the lateralization of the patella should also be restored.

Tajima et al. [14] studied the patellofemoral joint contact area and pressure after ACL reconstruction and concluded that DB ACL reconstruction restores the patellofemoral joint contact area and contact pressures better than SB ACL reconstruction. Several studies have also demonstrated that anatomic DB ACL reconstruction can restore the anterior tibial translation and rotational stability of the tibiofemoral joint more effectively than SB ACL reconstruction [1,4,6,14,26,27,31-33]. This should also be observed when improving patellofemoral kinematics, as the patellofemoral joint is linked to the tibiofemoral joint closely.

There were no relevant literatures about TT-TG values for different ACL reconstruction technologies. Goutallier et al. [23] proposed to measure the TT-TG between the most anterior point of the tibial tuberosity and the deepest bony point of the trochlear groove using perpendicular lines to the tangent of the bony borders of the posterior condyles on axial CT scans. Dejour et al. [28] measured the normal mean value of the TT-TG to be $12.7 \mathrm{~mm}$, which agrees with our finding of the intact knee with a mean TT-TG of $12.41 \pm 2.21 \mathrm{~mm}$. The golden standard method for measuring TT-TG distance is by CT scan [28]. However, the preoperative TT-TG values were measured from the patients' preoperative MRI to prevent patients from X-ray exposure within a short interval. Schoettle et al. [24] demonstrated that CT scan measurements were highly correlated to MRI scan ones of the TT-TG, with an inter-method reliability of $86 \%$ and an inter-person reliability of $82 \%$. Patients with patellofemoral disorder or patellar dislocation were excluded from this study to omit the possible effects of abnormal anatomy.

\section{Conclusion}

The enlarged TT-TG values from a ruptured ACL were significantly restored after ACL reconstruction. However, the TT-TG values after SB reconstruction were still significantly larger than in the intact knees. 
No significant difference was found between the postoperative TT-TG of the DB group and the intact knee. The native TT-TG value of the knee was much closer to that after DB reconstruction. Therefore, in order to restore patellofemoral joint stability better, avoiding the occurrence of osteoarthritis caused by patellofemoral cartilage injury after operation, we prefer anterior cruciate ligament double-bundle reconstruction as the choice of clinical operation.

\section{Acknowledgments}

This work was supported by the National Natural Science Foundation of China (Grant no. 81560374), and the Inner Mongolia Autonomous Region Natural Science Foundation of China (Grant nos. 2016MS0880, 2017MS08136, 2017MS08149, 2017MS08142 and 2018BS08002). We thank the American Journal Experts for their language editing, which greatly improved the manuscript.

\section{Conflict of interest}

None to report.

\section{References}

[1] Fu FH, Bennett CH, Lattermann C, Ma CB. Current trends in anterior cruciate ligament reconstruction. Part 1: Biology and biomechanics of reconstruction. The American Journal of Sports Medicine 1999; 27(6): 821-830. doi: $10.1177 / 03635465990270062501$.

[2] Lam MH, Fong DT, Yung P, Ho EP, Chan WY, Chan KM. Knee stability assessment on anterior cruciate ligament injury: Clinical and biomechanical approaches. Sports Medicine, Arthroscopy, Rehabilitation, Therapy \& Technology: SMARTT 2009; 1(1): 20. doi: 10.1186/1758-2555-1-20.

[3] Muneta T, Koga H, Mochizuki T, Ju YJ, Hara K, Nimura A, et al. A prospective randomized study of 4-strand semitendinosus tendon anterior cruciate ligament reconstruction comparing single-bundle and double-bundle techniques. Arthroscopy: the journal of arthroscopic \& related surgery: official publication of the Arthroscopy Association of North America and the International Arthroscopy Association 2007; 23(6): 618-628. doi: 10.1016/j.arthro.2007.04.010.

[4] Yagi M, Kuroda R, Nagamune K, Yoshiya S, Kurosaka M. Double-bundle ACL reconstruction can improve rotational stability. Clinical Orthopaedics and Related Research 2007; 454: 100-107. doi: 10.1097/BLO.0b013e31802ba45c.

[5] Yu JK, Ao YF, Yu CL, Luo H, Gong X, Chen LX, et al. Anatomic and clinical study on 4-bone-tunnel double-bundle anterior cruciate ligament. Zhonghua Yi Xue Za Zhi 2009; 89(29): 2019-2024.

[6] Zantop T, Petersen W, Sekiya JK, Musahl V, Fu FH. Anterior cruciate ligament anatomy and function relating to anatomical reconstruction. Knee Surgery, Sports Traumatology, Arthroscopy: Official Journal of the ESSKA 2006; 14(10): 982-992. doi: 10.1007/s00167-006-0076-z.

[7] Yu JK. [Comparative study on the clinic effects of arthroscopic reconstructions of single-bundle and double-bundle anterior cruciate ligament]. Zhongguo Gu Shang = China Journal of Orthopaedics and Traumatology 2016; 29(5): 391396.

[8] Biau DJ, Tournoux C, Katsahian S, Schranz P, Nizard R. ACL reconstruction: a meta-analysis of functional scores. Clinical Orthopaedics and Related Research 2007; 458: 180-187. doi: 10.1097/BLO.0b013e31803dcd6b.

[9] Gabriel MT, Wong EK, Woo SL, Yagi M, Debski RE. Distribution of in situ forces in the anterior cruciate ligament in response to rotatory loads. Journal of Orthopaedic Research: Official Publication of the Orthopaedic Research Society 2004; 22(1): 85-89. doi: 10.1016/s0736-0266(03)00133-5.

[10] Georgoulis AD, Ristanis S, Chouliaras V, Moraiti C, Stergiou N. Tibial rotation is not restored after ACL reconstruction with a hamstring graft. Clinical Orthopaedics and Related Research 2007; 454: 89-94. doi: 10.1097/BLO.0b013 e31802b4a0a.

[11] Tashman S, Collon D, Anderson K, Kolowich P, Anderst W. Abnormal rotational knee motion during running after anterior cruciate ligament reconstruction. The American Journal of Sports Medicine 2004; 32(4): 975-983. doi: $10.1177 / 0363546503261709$. 
[12] Woo SL, Kanamori A, Zeminski J, Yagi M, Papageorgiou C, Fu FH. The effectiveness of reconstruction of the anterior cruciate ligament with hamstrings and patellar tendon. A cadaveric study comparing anterior tibial and rotational loads. The Journal of Bone and Joint Surgery American Volume 2002; 84-a(6): 907-914.

[13] Stergiou N, Ristanis S, Moraiti C, Georgoulis AD. Tibial rotation in anterior cruciate ligament (ACL)-deficient and ACL-reconstructed knees: a theoretical proposition for the development of osteoarthritis. Sports Medicine (Auckland, NZ) 2007; 37(7): 601-613.

[14] Tajima G, Iriuchishima T, Ingham SJ, Shen W, van Houten AH, Aerts MM, et al. Anatomic double-bundle anterior cruciate ligament reconstruction restores patellofemoral contact areas and pressures more closely than nonanatomic single-bundle reconstruction. Arthroscopy: The journal of arthroscopic \& related surgery: official publication of the Arthroscopy Association of North America and the International Arthroscopy Association 2010; 26(10): 1302-1310. doi: 10.1016/j.arthro.2010.01.034.

[15] Kellgren JH, Lawrence JS. Radiological assessment of osteo-arthrosis. Annals of the Rheumatic Diseases 1957; 16(4): 494-502.

[16] Beighton P, Horan F. Orthopaedic aspects of the Ehlers-Danlos syndrome. The Journal of Bone and Joint Surgery British Volume 1969; 51(3): 444-453.

[17] Harner CD, Baek GH, Vogrin TM, Carlin GJ, Kashiwaguchi S, Woo SL. Quantitative analysis of human cruciate ligament insertions. Arthroscopy: The Journal of Arthroscopic \& Related Surgery: Official Publication of the Arthroscopy Association of North America and the International Arthroscopy Association 1999; 15(7): 741-749.

[18] Staubli HU, Rauschning W. Tibial attachment area of the anterior cruciate ligament in the extended knee position. Anatomy and cryosections in vitro complemented by magnetic resonance arthrography in vivo. Knee surgery, sports traumatology, arthroscopy: Official Journal of the ESSKA 1994; 2(3): 138-146.

[19] Gong X, Jiang D, Wang YJ, Wang J, Ao YF, Yu JK. Second-look arthroscopic evaluation of chondral lesions after isolated anterior cruciate ligament reconstruction: single-versus double-bundle reconstruction. The American Journal of Sports Medicine 2013; 41(10): 2362-2367. doi: 10.1177/0363546513496064.

[20] Leung KK, Yu JK, Luo H, Ao YF, Wang JQ, Cui GQ, et al. The position of the posterolateral bundle femoral tunnel during arthroscopic double-bundle anterior cruciate ligament reconstruction: a cadaveric study. Arthroscopy: the Journal of Arthroscopic \& Related Surgery: Official Publication of the Arthroscopy Association of North America and the International Arthroscopy Association 2011; 27(7): 959-964. doi: 10.1016/j.arthro.2011.02.023.

[21] Miao Y, Yu JK, Ao YF, Zheng ZZ, Gong X, Leung KK. Diagnostic values of 3 methods for evaluating meniscal healing status after meniscal repair: comparison among second-look arthroscopy, clinical assessment, and magnetic resonance imaging. The American Journal of Sports Medicine 2011; 39(4): 735-742. doi: 10.1177/0363546510388930.

[22] Miao Y, Yu JK, Zheng ZZ, Yu CL, Ao YF, Gong X, et al. MRI signal changes in completely healed meniscus confirmed by second-look arthroscopy after meniscal repair with bioabsorbable arrows. Knee Surgery, Sports Traumatology, Arthroscopy: Official Journal of the ESSKA 2009; 17(6): 622-630. doi: 10.1007/s00167-009-0728-x.

[23] Goutallier D, Bernageau J, Lecudonnec B. [The measurement of the tibial tuberosity. Patella groove distanced technique and results (author's transl)]. Revue de Chirurgie Orthopedique et Reparatrice de l'appareil Moteur 1978; 64(5): 423-428.

[24] Schoettle PB, Zanetti M, Seifert B, Pfirrmann CW, Fucentese SF, Romero J. The tibial tuberosity-trochlear groove distance; a comparative study between CT and MRI scanning. The Knee 2006; 13(1): 26-31. doi: 10.1016/j.knee.2005. 06.003.

[25] Brown CH, Jr., Carson EW. Revision anterior cruciate ligament surgery. Clinics in Sports Medicine 1999; 18(1): 109171.

[26] Georgoulis AD, Ristanis S, Moraiti CO, Paschos N, Zampeli F, Xergia S, et al. ACL injury and reconstruction: Clinical related in vivo biomechanics. Orthopaedics \& Traumatology, Surgery \& Research: OTSR 2010; 96(8 Suppl): S119-128. doi: 10.1016/j.otsr.2010.09.004.

[27] Morimoto Y, Ferretti M, Ekdahl M, Smolinski P, Fu FH. Tibiofemoral joint contact area and pressure after single- and double-bundle anterior cruciate ligament reconstruction. Arthroscopy: The Journal of Arthroscopic \& Related surgery: Official Publication of the Arthroscopy Association of North America and the International Arthroscopy Association 2009; 25(1): 62-69. doi: 10.1016/j.arthro.2008.08.014.

[28] Dejour H, Walch G, Nove-Josserand L, Guier C. Factors of patellar instability: an anatomic radiographic study. Knee Surgery, Sports Traumatology, Arthroscopy: Official Journal of the ESSKA 1994; 2(1): 19-26.

[29] Hsieh CH, Chen JC. Acute dislocation of the proximal tibiofibular joint. The Journal of Orthopaedic and Sports Physical Therapy 2009; 39(11): 826. doi: 10.2519/jospt.2009.0414.

[30] Shin CS, Chaudhari AM, Andriacchi TP. Valgus plus internal rotation moments increase anterior cruciate ligament strain more than either alone. Medicine and Science in Sports and Exercise 2011; 43(8): 1484-1491. doi: 10.1249/MSS. 0b013e31820f8395.

[31] Van de Velde SK, Bingham JT, Hosseini A, Kozanek M, DeFrate LE, Gill TJ, et al. Increased tibiofemoral cartilage contact deformation in patients with anterior cruciate ligament deficiency. Arthritis and Rheumatism 2009; 60(12): 36933702. doi: 10.1002/art.24965. 
[32] Belisle AL, Bicos J, Geaney L, Andersen MH, Obopilwe E, Rincon L, et al. Strain pattern comparison of doubleand single-bundle anterior cruciate ligament reconstruction techniques with the native anterior cruciate ligament. Arthroscopy: The Journal of Arthroscopic \& Related Surgery: Official Publication of the Arthroscopy Association of North America and the International Arthroscopy Association 2007; 23(11): 1210-1217. doi: 10.1016/j.arthro. 2007.06.021.

[33] Ho JY, Gardiner A, Shah V, Steiner ME. Equal kinematics between central anatomic single-bundle and double-bundle anterior cruciate ligament reconstructions. Arthroscopy: The Journal of Arthroscopic \& Related Surgery: Official Publication of the Arthroscopy Association of North America and the International Arthroscopy Association 2009; 25(5): 464-472. doi: 10.1016/j.arthro.2009.02.013. 